\title{
A CLOSED LOOP APPROACH TO TANK REACTOR MODEL SIMPLIFICATION
}

\author{
Veronica Olesen, Torsten Wik and Claes Breitholtz
}

\author{
Control and Automation Laboratory, Chalmers University of \\ Technology, Göteborg, Sweden \\ e-mail:veronica@s2.chalmers.se,tw@s2.chalmers.se, \\ cb@s2.chalmers.se
}

\begin{abstract}
Stirred tank reactors are widely used in the chemical process industry and in many of them there are problems and constraints related to the temperature control. In order to achieve a model suitable for temperature control of such a system a number of ad hoc assumptions and approximations are generally made. Here, a method to systematically evaluate the validity of such simplifications based on the resulting performance of the controlled closed loop system is proposed. The method is applied to a twocompartment model of a reactor with an exothermic reaction. It is then shown that many standard assumptions are reasonable but some assumptions are clearly not advisable because of possibly deteriorated closed loop performance. Copyright ${ }^{\circledR} 2005$ IFAC
\end{abstract}

Keywords: Non-Ideally Stirred Tank Reactor, Compartmental Model, Sensitivity Functions, Model Simplification

\section{INTRODUCTION}

An exothermic reaction in a reactor can easily run out of control, since a rise in temperature will make the reaction go faster and the reaction itself produces heat. To avoid instability many of these reactions are run today with highly diluted components and reaction temperatures well below optimal operating temperatures. Thus, an efficient temperature control would increase the productivity of such processes, since the reactant concentration and operating temperature can be higher. In order to determine a non-trivial temperature controller, reactor models suitable for controller design are required. Preferably, the models should be of low order, but with variables still having a direct physical interpretation.

There are many tank reactor models suitable for investigation of special phenomena or specific control strategies, see for instance (Lagerberg and Breitholtz, 1997; Abel and Marquardt, 2003) and references therein. However, in many of these models, assumptions have been made that might not be valid in general. For example, one commonly used approximation in tank reactor modelling is to ignore the temperature dependence of the specific heat capacities. In many models all heat capacities are also set to the heat capacity of water when water is used as liquid solvent. There are also many ways to reduce and simplify a complex first principles model, for example by nonlinear identification as described by Vargas and Allgöwer (2004). In this paper, we begin the modelling and model reduction with a detailed compartmental model of a non-ideally stirred tank reactor and then we propose a method to systematically evaluate the validity of such simplifications based on the resulting performance of the controlled closed loop system. The key steps are (i) a linearization of the compartmental model, (ii) a preliminary controller design for the simplified and for the detailed model, (iii) determination of the closed loop sensitivity functions and stability measures, and (iv) an evaluation of the deviation of these closed loop measures resulting from the model simplifications. 
Since not all tank reactors are perfectly mixed, we have used compartmental modelling to describe a nonideally stirred tank reactor. Compartmental modelling is used in many different applications, see for instance (Godfrey, 1983; Reuss and Bajpai, 1991). Here, the tank reactor is divided into smaller parts where each one of them is described as an ideally stirred tank reactor. How well stirred the total reactor is can then be described by fictitious flows between the compartments. As indicated by ten Cate et al. (2000) the compartmental relationships are hard to determine experimentally but can be found through computational fluid dynamics (CFD) calculations. The more compartments used, the better the model can describe the non-ideally stirred tank reactor. However, as a start, and since the method is easier to follow, we limit our study to a two-compartments model, which in many cases is sufficient to describe the non-ideality of the mixing (Alexopoulos et al., 2002). The dynamics of the cooling system have not been treated here, since the main focus has been on simplifications of the chemical reaction model. However, for controller design a model of the cooling system may be needed as well. An example model of a cooling system can be found in (Lagerberg and Breitholtz, 1997).

\section{SYSTEM DESCRIPTION}

\subsection{A Two-Compartments Model}

In the reactor model, the compartments are numbered 1 and 2 (see Figure 1), where the inlet and the outlet from the tank are located in compartment 1 . The reactor is equipped with a cooling jacket around the reactor, i.e. in compartment 1. A similar reactor is found in (Luyben, 1990). In this example, the dynamics of the cooling system are neglected and its temperature is considered as an operational degree of freedom.

The following reaction is assumed to take place in each of the two compartments of the tank reactor.

$$
A \stackrel{r}{\rightarrow} B
$$

where the reaction rate is described by

$$
r=e^{-\frac{E}{R T}} c_{A} k_{0}
$$

All symbols used in these and the following equations are listed in Section 5.

The densities $\rho$ of all compounds considered are assumed to be constant and all heat capacities $c_{p}$ are functions of temperature approximated by:

$$
\begin{aligned}
c_{p l}(T) & =\beta_{0 l}+\beta_{1 l} T+\beta_{2 l} T^{2} \\
c_{p A}(T) & =\beta_{0 A}+\beta_{1 A} T \\
c_{p B}(T) & =\beta_{0 B}+\beta_{1 B} T
\end{aligned}
$$

where $l$ denotes the liquid solvent.

Mass balances and energy balances of the two compartments then give the following nonlinear differential equations:

$$
\begin{aligned}
& \frac{d c_{A 1}}{d t}=\frac{1}{V_{1}}\left(Q_{f}\left(c_{A f}-c_{A 1}\right)+R_{21}\left(c_{A 2}-c_{A 1}\right)\right) \\
& -c_{A 1} k_{0} e^{-\frac{E}{R T_{1}}} \\
& \frac{d c_{A 2}}{d t}=\frac{1}{V_{2}} R_{12}\left(c_{A 1}-c_{A 2}\right)-c_{A 2} k_{0} e^{-\frac{E}{R T_{2}}} \\
& \frac{d c_{B 1}}{d t}=\frac{1}{V_{1}}\left(Q_{f}\left(c_{B f}-c_{B 1}\right)+R_{21}\left(c_{B 2}-c_{B 1}\right)\right) \\
& +c_{A 1} k_{0} e^{-\frac{E}{R T_{1}}} \\
& \frac{d c_{B 2}}{d t}=\frac{1}{V_{2}} R_{12}\left(c_{B 1}-c_{B 2}\right)+c_{A 2} k_{0} e^{-\frac{E}{R T_{2}}} \\
& \frac{d}{d t}\left(\int_{T_{0}}^{T_{1}}\left(V_{1}\left(c_{A 1} c_{p, A}+c_{B 1} c_{p, B}+c_{l 1} c_{p, l}\right)\right) d \tau\right. \\
& \left.+V_{1}\left(c_{A 1} h_{A}+c_{B 1} h_{B}+c_{l 1} h_{l}+h_{s 1}\right)\right) \\
& =Q_{f} \int_{T_{0}}^{T_{f}}\left(c_{A f} c_{p, A}+c_{B f} c_{p, B}+c_{l f} c_{p, l}\right) d \tau \\
& +Q_{f}\left(c_{A f} h_{A}+c_{B f} h_{B}+c_{l f} h_{l}+h_{s f}\right) \\
& +R_{21} \int_{T_{0}}^{T_{2}}\left(c_{A 2} c_{p, A}+c_{B 2} c_{p, B}+c_{l 2} c_{p, l}\right) d \tau \\
& +R_{21}\left(c_{A 2} h_{A}+c_{B 2} h_{B}+c_{l 2} h_{l}+h_{s 2}\right) \\
& -\left(Q_{o}+R_{12}\right) \int_{T_{0}}^{T_{2}}\left(c_{A 1} c_{p, A}+c_{B 1} c_{p, B}+c_{l 1} c_{p, l}\right) d \tau \\
& -\left(Q_{o}+R_{12}\right)\left(c_{A 1} h_{A}+c_{B 1} h_{B}+c_{l 1} h_{l}+h_{s 1}\right) \\
& +k_{0} e^{-\frac{E}{R T_{1}}} c_{A 1} V_{1}\left(-\Delta H_{r}\right)+P
\end{aligned}
$$

$$
\begin{aligned}
& \frac{d}{d t}\left(\int_{T_{0}}^{T_{2}} V_{2}\left(c_{A 2} c_{p, A}+c_{B 2} c_{p, B}+c_{l 2} c_{p, l}\right) d \tau\right. \\
& \left.\quad+V_{2}\left(c_{A 2} h_{A}+c_{B 2} h_{B}+c_{l 2} h_{l}+h_{s 2}\right)\right) \\
& =R_{12} \int_{T_{0}}^{T_{1}}\left(c_{A 1} c_{p, A}+c_{B 1} c_{p, B}+c_{l 1} c_{p, l}\right) d \tau \\
& \quad+R_{12}\left(c_{A 1} h_{A}+c_{B 1} h_{B}+c_{l 1} h_{l}+h_{s 1}\right) \\
& \quad-R_{21} \int_{T_{0}}^{T_{2}}\left(c_{A 2} c_{p, A}+c_{B 2} c_{p, B}+c_{l 2} c_{p, l}\right) d \tau \\
& \quad-R_{21}\left(c_{A 2} h_{A}+c_{B 2} h_{B}+c_{l 2} h_{l}+h_{s 2}\right) \\
& \quad+k_{0} e^{-\frac{E}{R T_{2}} c_{A 2} V_{2}\left(-\Delta H_{r}\right)}
\end{aligned}
$$



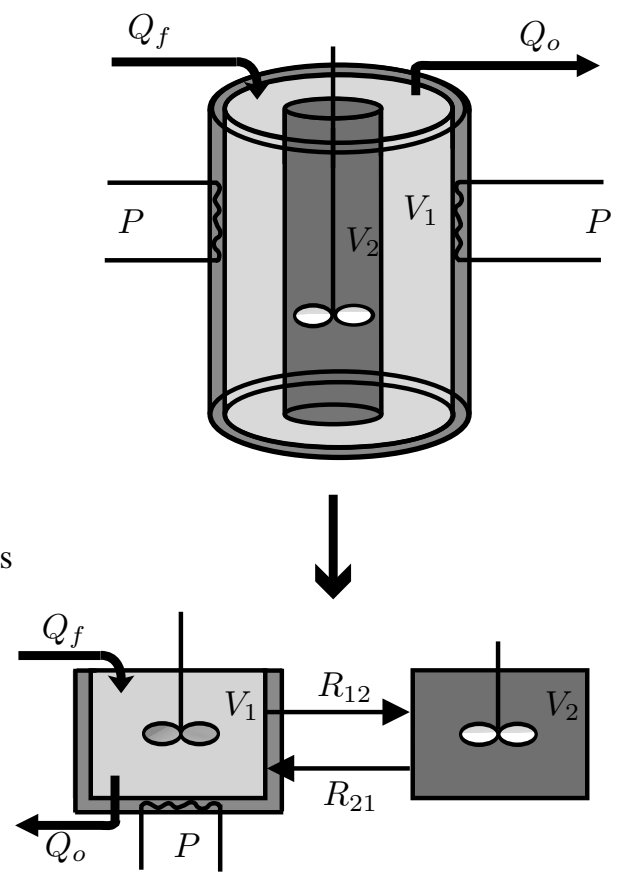

Fig. 1. An example of a two compartmental model

The concentration in compartment $i$ of the liquid solvent follows from an algebraic equation:

$$
c_{l i}=\frac{\rho_{l}}{M_{l}}\left(1-c_{A i} \frac{M_{A}}{\rho_{A}}-c_{B i} \frac{M_{B}}{\rho_{B}}\right)
$$

\subsection{Operating Window}

The continuous reactor can operate in a steady state under the right circumstances. In order to find the operating points for the two compartmental continuous reactor, the time derivatives of Equations (4) to (9) are set to zero. However for certain combinations of the variables $Q_{f}=Q_{o}, T_{f}, V_{1}, V_{2}, c_{A f}, c_{B f}, T_{1}$ and $T_{2}$ the continuous system cannot operate in a steady state, since either $c_{A 1}, c_{A 2}$ or $R_{12}=R_{21}$ would have to be negative then.

All points where the continuous system can reach a steady state define the operating window. Standard linearization around the operating point, results in a sixth order linearized model for each operating point in the operating window.

\subsection{A Linear Time Invariant State Space Model of Minimal Order}

If the cooling energy flow in compartment 1 is used as input and one of the two temperatures as output, the linearization results in two different linear models, each one of them having the same six states but different output signals. However, an observability and controllability analysis shows that two states or dynamic modes are uncontrollable.

With some chemical engineering insight, it can be seen why the system is not fully controllable. Since the chemical substances $A$ and $B$ are coupled through the chemical reaction, $c_{A i}$ and $c_{B i}$ can not be controlled separately. Every mole of $A$ disappearing due to the chemical reaction becomes $B$. The only way to control $c_{A i}$ and $c_{B i}$ separately is to change the concentration of $A$ or $B$ in the inflow. Hence, all state variables cannot be controlled using the external heat flow as the only control signal.

Since the linear state space model is not controllable, the state space realisation is not minimal. Hence the state space model can be reduced by one state per compartment to a forth order system. Expressing the sums $c_{A 1}+c_{B 1}$ and $c_{A 2}+c_{B 2}$ from the nonlinear state equations results in a linear subsystem

$$
\begin{aligned}
\frac{d}{d t}\left[\begin{array}{l}
c_{A 1}+c_{B 1} \\
c_{A 2}+c_{B 2}
\end{array}\right]= & \overbrace{\left[\begin{array}{cc}
-\frac{Q_{f}+R_{21}}{V_{1}} & \frac{R_{21}}{V_{1}} \\
\frac{R_{12}}{V_{2}} & -\frac{R_{12}}{V_{2}}
\end{array}\right]}^{\mathbf{A}_{2}}\left[\begin{array}{l}
c_{A 1}+c_{B 1} \\
c_{A 2}+c_{B 2}
\end{array}\right] \\
& +\left[\begin{array}{c}
\frac{Q_{f}}{V_{1}} \\
0
\end{array}\right]\left(c_{A f}+c_{B f}\right)
\end{aligned}
$$

The nonlinear differential equations of $c_{A 1}, c_{A 2}, H_{1}$ and $H_{2}$ can now be rewritten using the two new state variables from (11). Since Equation (11) is not dependant on the input signal $P$ or the other state variables, $c_{A 1}+c_{B 1}$ and $c_{A 2}+c_{B 2}$ describe the noncontrollable modes. Before the model can be reduced, the stability of these modes has to be determined. No stability problems can arise from the reduction if (11) is input output stable from $c_{A f}+c_{B f}$, i.e. $\mathbf{A}_{2}$ has both its eigenvalues in the negative half of the complex plane.

The eigenvalues $(\lambda)$ of $\mathbf{A}_{2}$ are found from:

$$
\begin{aligned}
0 & =\operatorname{det}\left(\lambda I-\mathbf{A}_{2}\right) \\
& =\lambda^{2}+\lambda\left(\frac{Q_{f}+R_{21}}{V_{1}}+\frac{R_{12}}{V_{2}}\right)+\frac{Q_{f}}{V_{1}} \frac{R_{12}}{V_{2}}(12)
\end{aligned}
$$

Both eigenvalues are in the negative half plane if and only if Equation (12) is Hurwitz, i.e. if and only if

$$
\frac{Q_{f}+R_{21}}{V_{1}}+\frac{R_{12}}{V_{2}}>0 \text { and } \frac{Q_{f}}{V_{1}} \frac{R_{12}}{V_{2}}>0
$$

This is true for all positive flows. If there is no inflow, i.e. $Q_{f}=0$, one of the eigenvalues will be zero. This will not cause a problem, since the system (11) lacks an input signal.

\section{ANALYSIS IN CLOSED LOOP}

Model errors will affect the sensitivity function $S=$ $(I+G F)^{-1}$ and the complementary sensitivity function $T \equiv I-S$ (Glad and Ljung, 2000). Since these 


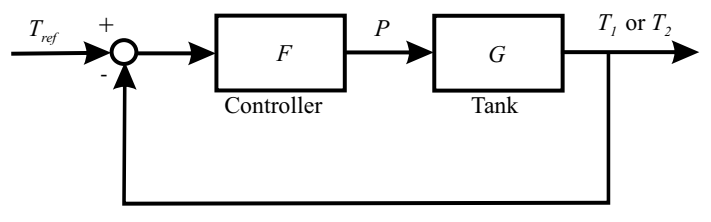

Fig. 2. Closed loop system

Table 1. Approximations and parameter changes tested

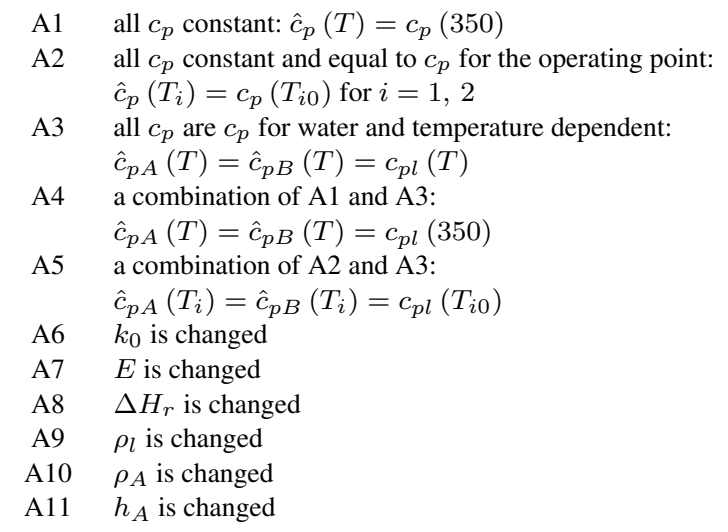

functions describe the performance of the controlled closed loop system, changes in $S$ and $T$ give an indication of the effect of the model change on the controlled system. $S$ and $T$ have been calculated for the closed loop in Figure 2, where the linearized single input single output (SISO) system $G$ is controlled by a controller $F$. The tank model $G$ is SISO with either $T_{1}$ or $T_{2}$ as output variable.

In order to evaluate the importance of parameter approximations and model assumptions the closed loop properties are studied for the situations given in Table 1 . For the resulting approximate model $\hat{G}$ a controller $\hat{F}$ is designed to give the system $\hat{G} \hat{F}$ designated closed loop characteristics. The controller $\hat{F}$ is then used in a closed loop with the original tank model $G$. From this closed loop system the corresponding sensitivity functions $\hat{S}$ and $\hat{T}$ are calculated. These altered sensitivity functions are then compared to $S$ and $T$ calculated for $G F$ in closed loop. In the original closed loop system $F$, is designed for $G$ in the same way as $\hat{F}$ is designed for $\hat{G}$.

\subsection{Choice of Controller}

A prerequisite for the analysis of sensitivity functions is a controller. The controller must achieve closed loop stability without being unnecessarily forgiving. A highly complex controller may well be best suited for controlling the system but in this investigation could also hide model errors important for a system controlled by a less complex controller. Hence, if a model error is small enough for the test controller to handle, other possible controllers should be able to handle that specific model error as well.
The tank model with $T_{1}$ as its output has a pole excess of one and if $T_{2}$ is chosen as output, the model has a pole excess of two. No time delays in either of the two systems are considered and all poles and zeros are located strictly in the negative half of the complex plane. Hence, the phase of the first system asymptotically reaches $-90^{\circ}$ as frequency increases and the argument of the second system reaches $-180^{\circ}$ asymptotically. Thus a simple integral (I) controller would give a stable closed loop system and also fulfill the prerequisite of a simple controller.

The I-controller used is designed to give the resulting feedback system for $G F$ and $\hat{G} \hat{F} 60^{\circ}$ phase margins.

\subsection{Closed Loop Measures}

If the integrals

$$
E_{S}=\int_{\log \omega_{1}}^{\log \omega_{2}}(\log |S|-\log |\hat{S}|)^{2} d \log \omega
$$

and

$$
E_{T}=\int_{\log \omega_{1}}^{\log \omega_{2}}(\log |T|-\log |\hat{T}|)^{2} d \log \omega
$$

are small the parameter change or approximation marginally affect the closed loop system and is therefore acceptable. However, the interpretation of a "small" value of $E_{S}$ and $E_{T}$ has to be defined for each system investigated. After simulation of the twocompartments tank model in closed loop with different approximate controllers $\hat{F}$, we conclude that the closed loop system will not be significantly affected if:

$$
E_{S}, E_{T}<80 \text { for } \omega_{1}=0, \omega_{2}=\infty
$$

and

$$
E_{S}, E_{T}<1 \quad \text { for } \omega_{1}=\omega_{c} / 2, \omega_{2}=2 \omega_{c}
$$

Here, significantly affected is defined as a noticeable difference in the step response of the nonlinear closed loop systems. The phase margin of the linear system $G \hat{F}$ in close loop is also investigated. That analysis serves as a measure of how the stability is affected by the parameter changes.

\subsection{Acceptable Model Changes}

The sensitivity and complementary sensitivity functions are compared to the original functions using Equations (14) and (15) for all approximations and parameter changes tested. The calculations are performed in a variety of operating points. As an example, the results for the operating point $T_{10}=315 \mathrm{~K}$ and $T_{20}=320 \mathrm{~K}$ are presented in Tables 2 and 3 for two different concentrations of $A$ in the inflow $\left(c_{A f}=5000 \mathrm{~mol} / \mathrm{m}^{3}\right.$ and $\left.c_{A f}=15000 \mathrm{~mol} / \mathrm{m}^{3}\right)$. 
Table 2. Error measures according to (14) and (15) for different approximations and integration limits $\omega_{1}$ and $\omega_{2}$ when the feed concentration $c_{A f}=5000 \mathrm{~mol} / \mathrm{m}^{3}$.
Table 3. Error measures according to (14) and (15) for different approximations and integration limits $\omega_{1}$ and $\omega_{2}$ when the feed concentration $c_{A f}=15000 \mathrm{~mol} / \mathrm{m}^{3}$.

\begin{tabular}{|c|c|c|c|c|}
\hline \multirow[t]{2}{*}{$\begin{array}{l}\text { approximation or } \\
\text { parameter change }\end{array}$} & \multicolumn{2}{|c|}{$\begin{array}{l}\omega_{1}=0 \\
\omega_{2}=\infty\end{array}$} & \multicolumn{2}{|c|}{$\begin{array}{l}\omega_{1}=\omega_{c} / 2, \\
\omega_{2}=2 \omega_{c}\end{array}$} \\
\hline & $E_{S}$ & $E_{T}$ & $E_{S}$ & $E_{T}$ \\
\hline & 10.7 & 3.22 & 0.0105 & 0.0167 \\
\hline & $5.9 \cdot 10^{-3}$ & $1.8 \cdot 10^{-3}$ & $6.1 \cdot 10^{-6}$ & $8.8 \cdot 10^{-6}$ \\
\hline & 57.9 & 17.5 & 0.0621 & 0.0870 \\
\hline & 58.6 & 17.7 & 0.0815 & 0.0599 \\
\hline 5 & 80.1 & 24.2 & 0.0855 & 0.118 \\
\hline$\hat{k}_{0}=0.9 k_{0}$ & 0.385 & 0.116 & $3.8 \cdot 10^{-4}$ & $6.1 \cdot 10^{-4}$ \\
\hline$\hat{k}_{0}=1.1 k_{0}$ & 1.33 & 0.402 & $1.3 \cdot 10^{-3}$ & $2.1 \cdot 10^{-3}$ \\
\hline$\hat{k}_{0}=0.5 k_{0}$ & 0.142 & 0.0430 & $1.4 \cdot 10^{-4}$ & $2.2 \cdot 10^{-4}$ \\
\hline$\hat{k}_{0}=2 k_{0}$ & 0.404 & 0.122 & $4.0 \cdot 10^{-4}$ & $6.4 \cdot 10^{-4}$ \\
\hline$\hat{k}_{0}=0.1 k_{0}$ & 0.255 & 0.0770 & $2.5 \cdot 10^{-4}$ & $4.0 \cdot 10^{-4}$ \\
\hline$\hat{k}_{0}=10 k_{0}$ & 4.66 & 1.41 & $4.6 \cdot 10^{-3}$ & $7.5 \cdot 10^{-3}$ \\
\hline$\hat{E}=0.99 E$ & 0.0708 & 0.0214 & $7.1 \cdot 10^{-5}$ & $1.1 \cdot 10^{-4}$ \\
\hline$\hat{E}=1.01 E$ & 0.0877 & 0.0265 & $8.8 \cdot 10^{-5}$ & $1.4 \cdot 10^{-4}$ \\
\hline$\hat{E}=0.97 E$ & 0.140 & 0.0422 & $1.4 \cdot 10^{-4}$ & $2.2 \cdot 10^{-4}$ \\
\hline$\hat{E}=1.03 E$ & 0.981 & 0.296 & $9.9 \cdot 10^{-4}$ & $1.5 \cdot 10^{-3}$ \\
\hline$\hat{E}=0.93 E$ & 0.936 & 0.283 & $9.4 \cdot 10^{-4}$ & $1.5 \cdot 10^{-3}$ \\
\hline$\hat{E}=1.07 E$ & 0.0204 & $6.1 \cdot 10^{-3}$ & $2.0 \cdot 10^{-5}$ & $3.1 \cdot 10^{-5}$ \\
\hline$\Delta \hat{H}_{r}=1.1 \Delta H_{r}$ & $1.3 \cdot 10^{-5}$ & $2.8 \cdot 10^{-6}$ & $1.2 \cdot 10^{-8}$ & $1.9 \cdot 10^{-8}$ \\
\hline$\Delta \hat{H}_{r}=0.9 \Delta H_{r}$ & $1.3 \cdot 10^{-5}$ & $2.8 \cdot 10^{-6}$ & $1.2 \cdot 10^{-8}$ & $1.9 \cdot 10^{-8}$ \\
\hline$\Delta \hat{H}_{r}=1.5 \Delta H_{r}$ & $3.1 \cdot 10^{-4}$ & $9.2 \cdot 10^{-5}$ & $3.1 \cdot 10^{-7}$ & $4.7 \cdot 10^{-7}$ \\
\hline$\Delta \hat{H}_{r}=0.5 \Delta H_{r}$ & $3.1 \cdot 10^{-4}$ & $9.2 \cdot 10^{-5}$ & $3.1 \cdot 10^{-7}$ & $4.7 \cdot 10^{-7}$ \\
\hline$\Delta \hat{H}_{r}=10 \Delta H_{r}$ & 0.0803 & 0.0242 & $8.06 \cdot 10^{-5}$ & $1.2 \cdot 10^{-4}$ \\
\hline$\Delta \hat{H}_{r}=0.1 \Delta H_{r}$ & $5.0 \cdot 10^{-4}$ & $1.5 \cdot 10^{-4}$ & $5.0 \cdot 10^{-7}$ & $7.7 \cdot 10^{-7}$ \\
\hline$\hat{\rho}_{l}=0.98 \rho_{l}$ & 7.36 & 2.20 & $7.4 \cdot 10^{-3}$ & 0.0111 \\
\hline$\hat{\rho}_{l}=1.05 \rho_{l}$ & 42.7 & 12.8 & 0.0457 & 0.0616 \\
\hline$\hat{\rho}_{A}=0.95 \rho_{A}$ & 8.26 & 2.48 & $8.3 \cdot 10^{-3}$ & 0.0125 \\
\hline$\hat{\rho}_{A}=1.07 \rho_{A}$ & 12.7 & 3.79 & 0.0133 & 0.0185 \\
\hline$\hat{\rho}_{B}=0.93 \rho_{B}$ & 0.0993 & 0.0297 & $1.0 \cdot 10^{-4}$ & $1.5 \cdot 10^{-4}$ \\
\hline$\hat{\rho}_{B}=1.06 \rho_{B}$ & 0.0529 & 0.0158 & $5.4 \cdot 10^{-5}$ & $7.9 \cdot 10^{-5}$ \\
\hline$\hat{h}_{A}=1.36 h_{A}$ & $6.5 \cdot 10^{-4}$ & $2.0 \cdot 10^{-4}$ & $6.7 \cdot 10^{-7}$ & $9.7 \cdot 10^{-7}$ \\
\hline$\hat{h}_{A}=0.90 h_{A}$ & $1.2 \cdot 10^{-5}$ & $2.6 \cdot 10^{-6}$ & $1.2 \cdot 10^{-8}$ & $1.7 \cdot 10^{-8}$ \\
\hline
\end{tabular}

The results are presented for the system where $T_{1}$ is output but qualitatively the results for $T_{2}$ are equivalent. Model changes can also affect the stability margins, and therefore the phase margins for $G \hat{F}$ in closed loop are calculated. The results from the analyses are presented more in detail in (Olesen, 2004).

For $c_{A f}=5000 \mathrm{~mol} / \mathrm{m}^{3}$ almost all approximations and parameter changes studied seem to be acceptable for the operating point chosen. However, if the concentrations of $A$ and $B$ are increased, the $c_{p}$ approximations $\mathrm{A} 3, \mathrm{~A} 4$ and $\mathrm{A} 5$, where $c_{p}$ for the liquid solvent is used as an overall $c_{p}$, will cause significant deterioration. From the calculations of $E_{S}$ and $E_{T}$ for all operating points and different values of $c_{A f}$ it turns out that, as a rule of thumb, the approximations A3, $\mathrm{A} 4$ and $\mathrm{A} 5$ should not be used if $c_{A}+c_{B} \geq c_{l} / 2$.

An interesting result is also that the approximation $\mathrm{A} 2$, where $c_{p}$ is assumed to be its value in the operating point, hardly causes any model error at all for the closed loop system in Table 2. However, a rise in concentration will affect this approximation negatively but the approximation is still useful. A further investigation of A2 shows that it is a better approximation the closer the two operating point temperatures in the compartments are.

\begin{tabular}{|c|c|c|c|c|}
\hline \multirow[t]{2}{*}{$\begin{array}{l}\text { approximation or } \\
\text { parameter change }\end{array}$} & \multicolumn{2}{|c|}{$\begin{array}{l}\omega_{1}=0 \\
\omega_{2}=\infty\end{array}$} & \multicolumn{2}{|c|}{$\begin{array}{l}\omega_{1}=\omega_{c} / 2, \\
\omega_{2}=2 \omega_{c}\end{array}$} \\
\hline & $E_{S}$ & $E_{T}$ & $E_{S}$ & $E_{T}$ \\
\hline 1 & 3.67 & 1.12 & $3.7 \cdot 10^{-3}$ & $5.8 \cdot 10^{-3}$ \\
\hline 12 & 92.0 & 28.1 & 0.0989 & 0.137 \\
\hline 3 & 2664 & 814 & 3.65 & 3.02 \\
\hline 4 & 2693 & 823 & 3.70 & 3.05 \\
\hline 15 & 2707 & 827 & 3.72 & 3.06 \\
\hline$\hat{k}_{0}=0.9 k_{0}$ & 0.470 & 0.143 & $4.7 \cdot 10^{-4}$ & $7.3 \cdot 10^{-4}$ \\
\hline$\hat{k}_{0}=1.1 k_{0}$ & 1.32 & 0.403 & $1.3 \cdot 10^{-3}$ & $2.1 \cdot 10^{-3}$ \\
\hline$\hat{k}_{0}=0.5 k_{0}$ & 0.0254 & $7.8 \cdot 10^{-3}$ & $2.6 \cdot 10^{-5}$ & $4.0 \cdot 10^{-5}$ \\
\hline$\hat{k}_{0}=2 k_{0}$ & 2.99 & 0.913 & $3.0 \cdot 10^{-3}$ & $4.7 \cdot 10^{-3}$ \\
\hline$\hat{k}_{0}=0.1 k_{0}$ & 0.780 & 0.239 & $7.9 \cdot 10^{-4}$ & $1.2 \cdot 10^{-3}$ \\
\hline$\hat{k}_{0}=10 k_{0}$ & 239.4 & 73.2 & 0.216 & 0.403 \\
\hline$\hat{E}=0.99 E$ & 5.72 & 1.75 & $5.7 \cdot 10^{-3}$ & $9.1 \cdot 10^{-3}$ \\
\hline$\hat{E}=1.01 E$ & 0.132 & 0.0402 & $1.3 \cdot 10^{-4}$ & $2.1 \cdot 10^{-4}$ \\
\hline$\hat{E}=0$ & 7.22 & 2.21 & $7.1 \cdot 10^{-3}$ & 0.0114 \\
\hline$\hat{E}=1.03 E$ & $5.6 \cdot 10^{-3}$ & $1.7 \cdot 10^{-3}$ & $5.6 \cdot 10^{-6}$ & $8.7 \cdot 10^{-6}$ \\
\hline$\hat{E}=0.93 E$ & 1.573 & 29.7 & 0.0914 & 0.159 \\
\hline$\hat{E}=1.07 E$ & 0.0204 & 0.481 & $1.6 \cdot 10^{-3}$ & $2.4 \cdot 10^{-3}$ \\
\hline$\Delta \hat{H}_{r}=1.1 \Delta H_{r}$ & 3.51 & 1.07 & $3.5 \cdot 10^{-3}$ & $5.6 \cdot 10^{-3}$ \\
\hline$\Delta \hat{H}_{r}=0.9 \Delta H_{r}$ & $2.6 \cdot 10^{-3}$ & $7.8 \cdot 10^{-4}$ & $2.6 \cdot 10^{-6}$ & $4.0 \cdot 10^{-6}$ \\
\hline$\Delta \hat{H}_{r}=1.5 \Delta H_{r}$ & 4.32 & 1.32 & $4.3 \cdot 10^{-3}$ & $6.8 \cdot 10^{-3}$ \\
\hline$\Delta \hat{H}_{r}=0.5 \Delta H_{r}$ & 0.0639 & 0.0195 & $6.5 \cdot 10^{-5}$ & $1.0 \cdot 10^{-4}$ \\
\hline$\Delta \hat{H}_{r}=10 \Delta H_{r}$ & 260.8 & 79.8 & 0.234 & 0.440 \\
\hline$\Delta \hat{H}_{r}=0.1 \Delta H_{r}$ & 0.167 & 0.0512 & $1.7 \cdot 10^{-4}$ & $2.6 \cdot 10^{-4}$ \\
\hline$\hat{\rho}_{l}=0.98 \rho_{l}$ & & 3.29 & 0.0107 & 0.0170 \\
\hline$\hat{\rho}_{l}=$ & 135.2 & 41. & 0.147 & 0.196 \\
\hline$\hat{\rho}_{A}=$ & 975 & 2970 & 4.23 & 21.3 \\
\hline$\hat{\rho}_{A}=$ & 6906 & 209 & 11.0 & 5.80 \\
\hline$\hat{\rho}_{B}=$ & 54.4 & 16.5 & & 0.0870 \\
\hline$\hat{\rho}_{B}=1.06 \rho_{B}$ & 33.3 & 10.1 & 0.0348 & 0.0499 \\
\hline$\hat{h}_{A}=1.36 h_{A}$ & 1.00 & 0.304 & $1.0 \cdot 10^{-3}$ & $1.5 \cdot 10^{-3}$ \\
\hline$\hat{h}_{A}=0.90 h_{A}$ & $3.8 \cdot 10^{-3}$ & $1.2 \cdot 10^{-3}$ & $5.9 \cdot 10^{-6}$ & $4.5 \cdot 10^{-7}$ \\
\hline
\end{tabular}

From a study of the phase margins, we find that approximations A3, A4 and A5 decrease the phase margin by $10^{\circ}$ for high concentrations of $A$ in the tank. This observation also indicates that the assumption has to be used with care.

As seen in Table 3, changes in density can have a large effect on the system. The high values of $E_{S}$ and $E_{T}$ for $\rho_{A}$ in Table 3 could be taken as an indication that a dependence of the densities on temperature may have to be considered for high concentrations, which requires a further investigation along similar lines.

Tables 2 and 3 both indicate that variations in $E, k_{0}$, $\Delta H_{r}$ and $h_{A}$ of the magnitudes presented in Tables 2 and 3 will not affect the closed loop. However, these variations affect the operating window.

To test the generality of the results, new systems $G$ have been constructed for other values of $E$ and $\Delta H$ and a higher temperature dependence of $c_{p}\left(\beta_{1 \lambda}\right.$ doubled). The conclusions drawn from the presented examples are valid for these new $G$ as well.

\section{CONCLUSIONS}

For compartmental models of chemical reactors, sensitivity analysis is a powerful tool for investigating how approximations and parameter changes will affect 
the controller design. However, suitable measures for the changes in sensitivity function $\left(E_{S}\right)$ and complementary sensitivity function $\left(E_{T}\right)$ have to be decided for each system.

From the analysis made on this example system it can be seen that the temperature dependance of $c_{p}$ can be ignored, especially if the constant $c_{p}$ value is chosen as $c_{p}$ in the operating point for each substance. To approximate $c_{p}$ as $c_{p}$ for the liquid solvent is not advisable if the solution is not highly diluted.

The exponential temperature dependence of the reaction rate (Equation (2)) cannot be replaced by a polynomial in this example, since even small changes in $E$ will strongly affect the operating window.

\section{LIST OF SYMBOLS}

$A$

$B \quad$ The product

$c_{\lambda} \quad$ Concentration of substance $\lambda=A, B, l$ $\left(\mathrm{mol} / \mathrm{m}^{3}\right)$

$c_{p, \lambda} \quad$ Heat capacity of substance $\lambda(\mathrm{J} /(\mathrm{mol} \cdot K))$

$E \quad$ Activation energy $(\mathrm{J} / \mathrm{mol})$

$H_{i} \quad$ Enthalpy in compartment $i$

$k_{0} \quad$ Reaction dependent coefficient $s^{-1}$

$l \quad$ Liquid solvent

$M_{\lambda} \quad$ Molar mass of component $\lambda(\mathrm{g} / \mathrm{mol})$

$P \quad$ External heat transferred to the system $(W)$

$Q_{f}, Q_{o} \quad$ Feed flow and outlet flow from the whole reactor $\left(\mathrm{m}^{3} / \mathrm{s}\right)$

$R_{i j} \quad$ Flow from compartment $i$ to compartment $j\left(\mathrm{~m}^{3} / \mathrm{s}\right)$

$r \quad$ Reaction rate $\left(\mathrm{mol} /\left(\mathrm{m}^{3} \cdot \mathrm{s}\right)\right)$

$R \quad$ The ideal gas constant $(\mathrm{J} /(\mathrm{mol} \cdot \mathrm{K}))$

$t \quad$ Time $(s)$

$T \quad$ Temperature $(K)$

$V_{i} \quad$ Volume of compartment i $\left(\mathrm{m}^{3}\right)$

$\beta_{k \lambda} \quad$ Coefficient $k$ in the temperature function of $c_{p}(T)$ for component $\lambda$

$\Delta H_{r} \quad$ Heat of reaction $(\mathrm{J} / \mathrm{mol})$

$\rho_{\lambda} \quad$ Density of component $\lambda\left(\mathrm{g} / \mathrm{m}^{3}\right)$

\section{NOMINAL PARAMETER VALUES}

$\begin{array}{ll}c_{A f} & 5000 \mathrm{or} 15000 \mathrm{~mol} / \mathrm{m}^{3} \\ c_{B f} & 100 \mathrm{~mol} / \mathrm{m}^{3} \\ E & 70000 \mathrm{~J} / \mathrm{mol} \\ k_{0} & 19.6 \cdot 10^{6} \mathrm{~s}^{-1} \\ M_{A} & 46 \cdot 10^{-3} \mathrm{~g} / \mathrm{mol} \\ M_{B} & 46 \cdot 10^{-3} \mathrm{~g} / \mathrm{mol} \\ M_{l} & 18 \cdot 10^{-3} \mathrm{~g} / \mathrm{mol} \\ Q_{f}, Q_{o} & 5 \cdot 10^{-3} \mathrm{~m}^{3} / \mathrm{s} \\ R & 8.314 \mathrm{~J} /(\mathrm{mol} \cdot \mathrm{K}) \\ V_{1} & 7 \mathrm{~m}^{3} \\ V_{2} & 3 \mathrm{~m}^{3}\end{array}$

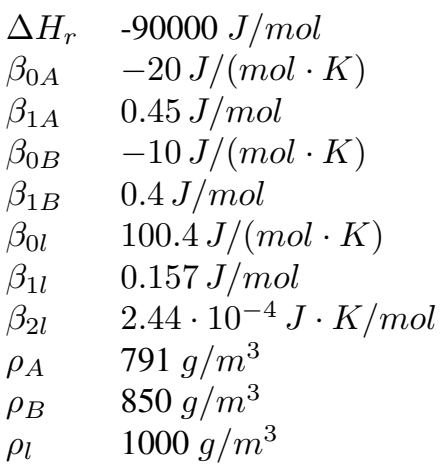

\section{REFERENCES}

Abel, O. and W. Marquardt (2003). Scenariointegrated on-line optimisation of batch reactors. Journal of Process Control 13(8), 703-715.

Alexopoulos, A.H., D. Maggioris and C. Kiparissides (2002). CFD analysis of turbulence non-homogeneity in mixing vessels a twocompartment model. Chemical Engineering Science 57(10), 1735-1752.

Glad, Torkel and Lennart Ljung (2000). Control Theory: Multivariable and Nonlinerar Methods. Taylor \& Francis, London.

Godfrey, Keith (1983). Compartmental Models and Their Application. Academic Press, London.

Lagerberg, Adam and Claes Breitholtz (1997). A study of gain scheduling control applied to an exothermic CSTR. Chemical Engineering \& Technology 20(7), 435-444.

Luyben, William L. (1990). Process Modeling, Simulation, and Control for Chemical Engineers. 2nd ed.. McGraw-Hill, inc., New York.

Olesen, Veronica (2004). Model reducion for nonideally stirred tank reactor processes - a closed loop perspective. Licentiate thesis. Technical Report 502L. Department of Signals and Systems, Chalmers University of Technology. Göteborg, Sweden.

Reuss, Matthias and Rakesh Bajpai (1991). Stirred tank models. In: Biotechnology IV: Measuring, modelling and control (Rehm and Reed, Eds.). Vol. 4. VCH, Weinheim. pp. 299-348.

ten Cate, A., S. K. Bermingham, J. J. Derksen and H. M. J. Kramer (2000). Compartmental modeling of an 1100L DTB crystallizer based on large eddy flow simulation. In: 10th European Conference on Mixing (H. E. A. van den Akker and J. J. Derksen, Eds.). pp. 255-264.

Vargas, Alejandro and Frank Allgöwer (2004). Model reduction for process control using iterative nonlinear identification. In: Proceedings of the 2004 American Control conference. Vol. 4. Boston, Massachusetts. pp. $2915-2920$. 